\title{
A Study on the Bead Geometry with Offset based on Image Processing Algorithm
}

\author{
Gang Zhang, Tae-Jong Yun, Won-Bin Oh, Bo-Ram Lee, Byeol Lim and Ill-Soo Kim \\ Department of Mechanical Engineering, Mokpo National University \\ 1666 Yeongsan-ro, Cheonggye-myeon, Muan-gun, Jeollanam-do, 58554, South Korea \\ zhanggang@mokpo.ac.kr; tjyun@mokpo.ac.kr; wboh@mokpo.ac.kr; boramess@mokpo.ac.kr; \\ zxzc1020@mokpo.ac.kr; ilsookim@mokpo.ac.kr
}

\begin{abstract}
During the robotic arc welding process, not only the images taken are often unclear, but also the features are not obvious due to the interference of arc, splash and smoke. Therefore, it is necessary to carefully develop the image process algorithm for the image processing to complete the weld inspection. In this study, the robotic GMA (Gas Metal Arc) welding has been applied to bead-on-plate welding with vertical position. The images with different offsets were obtained based on infrared camera through experiments which might be obtained, and a set of image processing algorithms was designed to detect the geometrical bead images according to the different offsets. In image processing procedure, the first step is to perform the pre-processing to improve the contrast of the image. Then, the edge of the image is detected by the canny operator. Finally, the centre curve equation to predict the bead geometry is obtained by the least square method as well as the angle size is obtained according to the slope of the equation. The bead geometry obtained by the image processing is compared with the bead geometry obtained from the experiment in order to verify the feasibility of the developed algorithm. The experimental results are clear that the error is small so that the developed algorithm can be employed in actual GMA welding process with robot.
\end{abstract}

Keywords: Robotic GMA welding, Bead geometry, Offset, Image process, Edge detection, Centreline extraction.

\section{Introduction}

GMA welding is one of the most important technologies in the fields of modern manufacturing. With the development of robot technology, a GMA welding process has been frequently increased the new requirements such as seam tracking system, automation, quality control system [1]. Recently, many researchers [2]-[3] has been focused on developing how to ensure the GMA welding quality, improve production efficiency and the working conditions, cut down the cost in manufacture, etc. Traditionally, human experts in welding processes perform quality control through visual inspection [2]. However, humans cannot do real-time monitor of the arc welding process when it is occurring since the radiation light emitted from the electrical arc was harmful for the eyes. Thus, an alternative is realizing the monitoring of arc welding process using machine vision techniques. Image processing technology is one of the key techniques in the robotic GMA welding. It makes directly whether the output control signal which could affect the seam tracking precision of the executive mechanism is right or not [3]. In recent years, peoples [4]-[5] have carried out research work in the field of automatic identification of weld seam track. Shao et al. [4] developed an image processing algorithm to analyze droplets contours. The algorithm was used to remove edges far from droplet common shape with a K-mean clustering algorithm. In order to improve the moving trajectory accuracy of the industrial robots which applied for robotic GMA welding process, Zhang [5] utilized a Gray Projecting Integral (GPI) transform method for seam recognition in trajectory tracking.

However, it might be required for overcoming the interference of the arc welding process to obtain a relatively clear image of the weld seam, and obtaining the center position of the weld by image processing due to the many disturbances in the welding process. Therefore, this paper is mainly concentrated on development of an optimal image processing algorithm based on infrared camera, which can accurately obtain the welding path and characteristic parameters to effectively control of seam tracking process and realize welding automation. The image processing process includes early noise removal, contrast enhancement, and late feature extraction. The binarized image is processed by the Canny operator for edge detection, and the least square method is employed to fit the weld edge. According to the equation fitted by the least squares method, the bead geometry and the position of the welding torch with the different offset can be obtained. The error between the 
obtained offset by calculating the image processing and the measured offset was performed to verify the reliability of the algorithm.

\section{Experimental Works}

An infrared camera was installed to align the torch with the line end to transmit thermal images in real time. Since the camera itself is cooled, no additional cooling system is installed. Not only protective quartz windows are made to protect the lens from smoke and splashes, but also other external environments were integrated for the robotic GMA welding process. Figure 1 shows the experimental setup for a robotic GMA welding system which included an infrared camera mounted in the torch part and a protective quartz window for the extraction of welded parts. Figure 2 represented a schematic diagram of a welding experiment for extraction of thermal image.

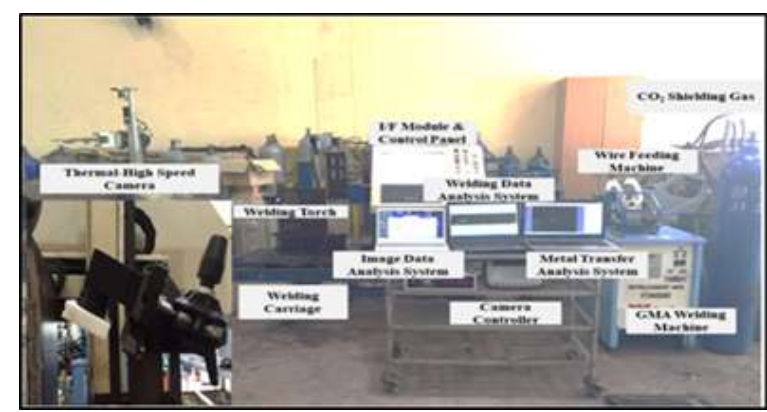

Fig. 1: Experimental setup for the robotic GMA welding process.

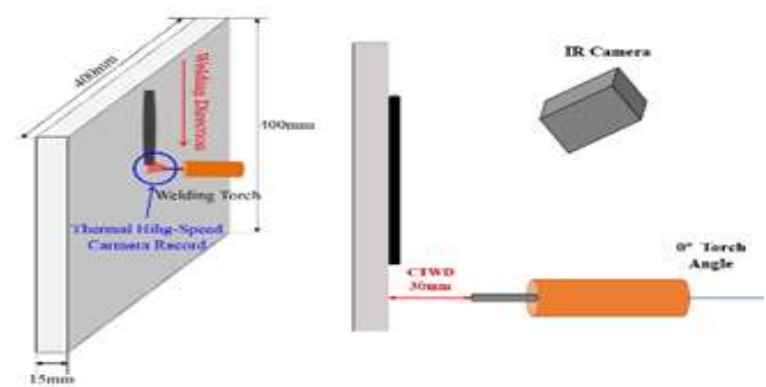

Fig. 2: A schematic diagram of GMA welding process for extraction of thermal image.

\section{The Image Processing for Optimal Welding Images}

\subsection{Image after pre-processing}

The purpose of the pre-processing image process is to remove the influence of interference points such as strong light and thermal deformation on the image taken during the robotic GMA welding process. It is worth of mention that the original image is in RGB (Red, Green, and Blue) model in which it should be converted to gray scale. In order to remove the noise, the performance of the median filter to process the image is superior. Histogram equalization method performs to further improve the contrast of thermal image well. The pre-processed results by current experimental apparatus were presented in figure 3. 


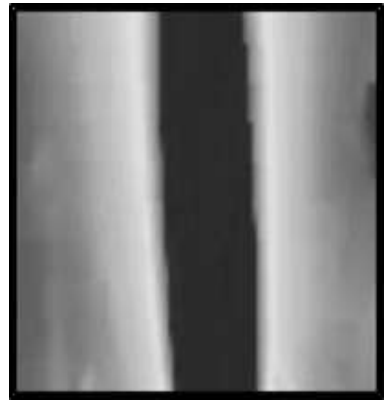

(a) Image

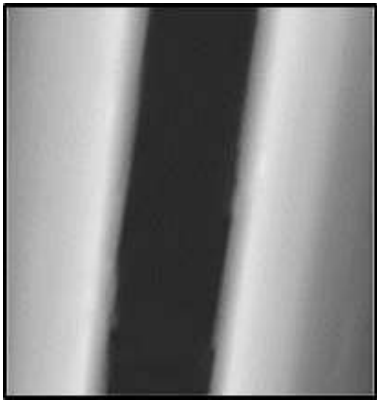

(b) Image

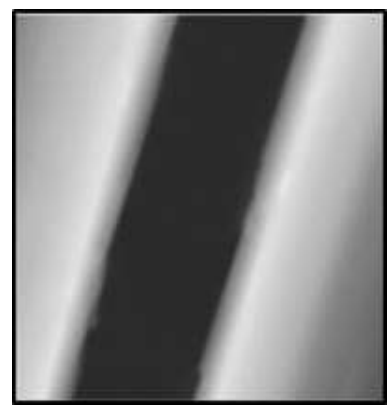

(c) Image

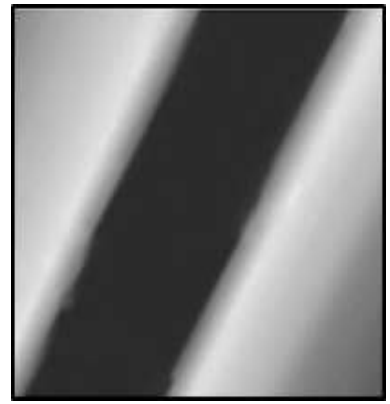

(d) Image

Fig. 3: The results of images after pre-processing.

\subsection{Edge detection and contour extraction}

The edge acquisition of the image is to replace the entire image information with the edge information that obtains most of the information of the image. The advantage of this is that the amount of data is greatly reduced, and the information which could be considered irrelevant is removed, and the essential structural information of the image is retained. The edge detection mainly employed the edge detection operator to enhance the edge of the image, and then performed threshold setting to extract the edge point information. The edge detection operator was employed the abrupt nature of the edge of the image to detect the edge. This study was employed Canny [6] operator for edge detection. It has the advantage of not losing important edges, no false edges, and minimal deviation between the actual edge and the detected edge position. The results for edge detection were shown in figure 4. In this study, the bead geometry is required image contour properties obtained using the Image Region Analyzer toolbox in Matlab software. According to the different properties of each contour, the extra contours were removed and obtained the image with only the bead geometry. The results were shown in figure 5.

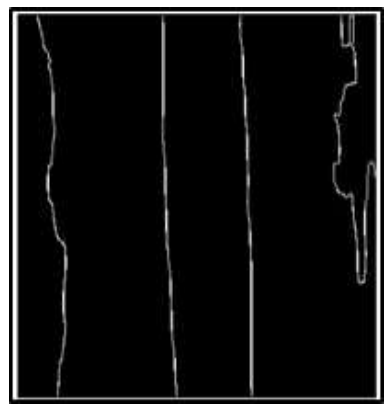

(a) Image

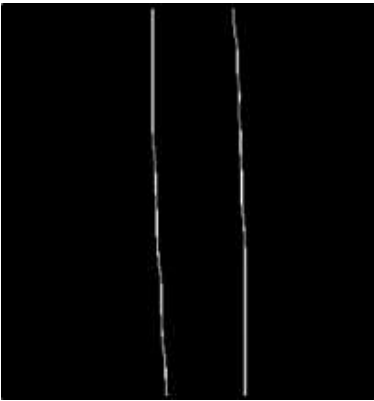

(a) Image

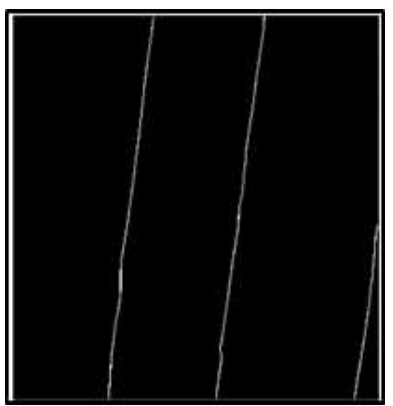

(b) Image

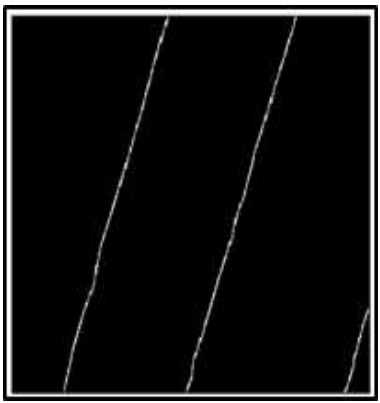

(c) Image

Fig. 4: Images after Canny operator for edge detection.

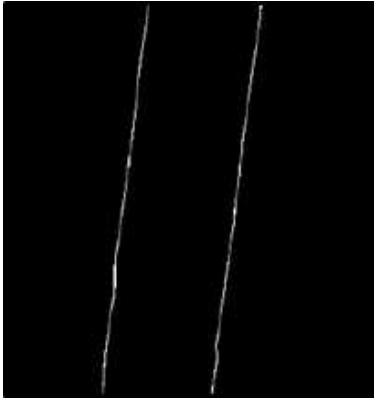

(b) Image

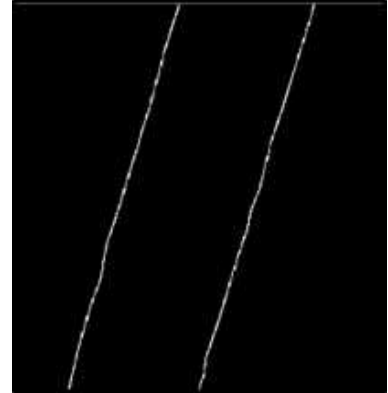

(c) Image

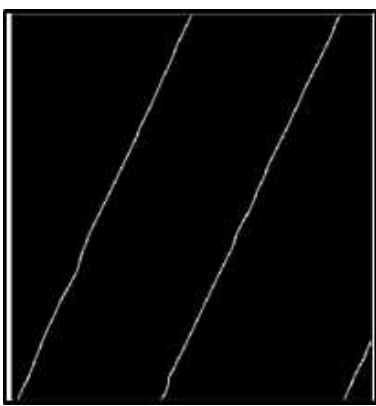

(d) Image

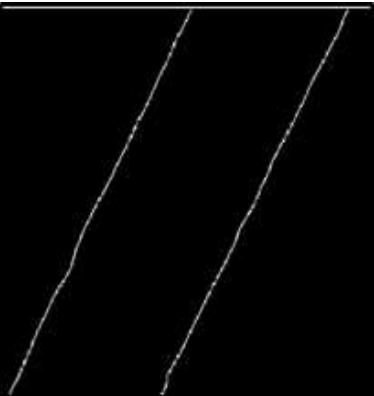

(d) Image

Fig. 5: The results of images after contour extraction. 


\subsection{Centreline extraction}

Since the edges of the refined image are not continuous, there are discrete and free pixels so that the free points be removed by curve fitting to connect the discrete pixels. For this purpose, this paper would be chosen the least squares method to fit the edge curve. Since the sample workpiece used in this paper is relatively neat, the edge of the up and groove is also basically symmetrical. The centreline can be extracted by averaging based on the least squares fitting on weld edge. The images after the extraction of the centreline were shown in figure 6 .

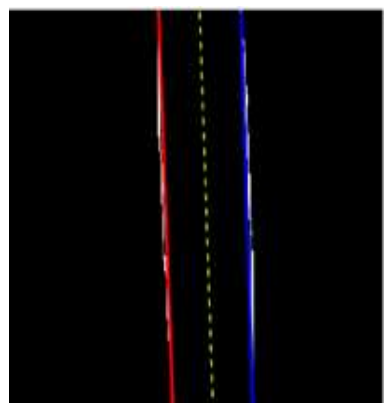

(a) Image

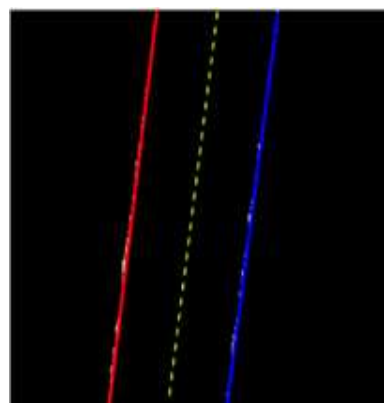

(b) Image

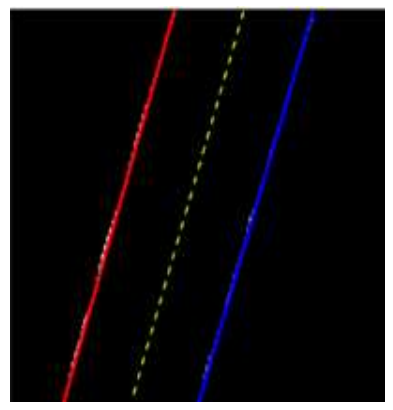

(c) Image

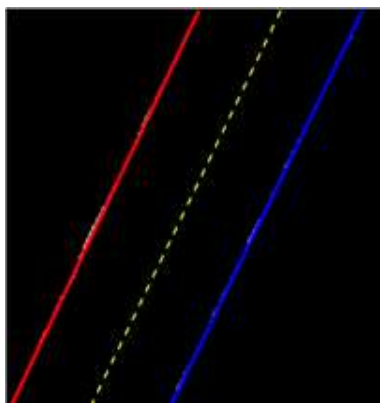

(d) Image

Fig. 6: Images after centreline extraction.

In order to verify the accuracy of the developed algorithm, the calculated offset of the bead geometry is compared to the measured offset. The equation of the central straight line is obtained by the least squares method, and the inclination angle of the straight line is calculated according to the slope of the equation. In fact, the actual offset is the complementary angle of the oblique angle. Table 1 represented the comparison between calculated and measured offsets. It can be concluded that maximum error between the calculated and the actual offsets is 2.1 degrees, which proves the accuracy of the developed algorithm.

Table 1: Comparison between the calculated and measured offsets.

\begin{tabular}{|l|l|l|l|l|l|}
\hline No. of Images & Slope constant & Inclination angle $\left(^{\circ}\right)$ & Calculated offset $\left(^{\circ}\right)$ & Measured offset $\left(^{\circ}\right)$ & Error $\left(^{\circ}\right)$ \\
\hline 1 & 26.64 & 87.850 & 2.150 & 0 & 2.150 \\
\hline 2 & 7.132 & 81.730 & 8.270 & 10 & 1.730 \\
\hline 3 & 3.069 & 71.952 & 18.004 & 20 & 1.996 \\
\hline 4 & 1.881 & 62.003 & 27.997 & 30 & 2.003 \\
\hline
\end{tabular}

\section{Conclusions}

This paper mainly analysed the image processing technology for seam tracking in the robotic GMA welding process. The digital images of the welded specimens captured were processed to improve the image quality for analysis using image processing toolbox of MATLAB. According to the purpose of vision-based weld inspection, a completed set of image processing algorithms are developed. By reasonably selecting the filtering, sharpening, edge detection, contour extraction, edge line fitting and other methods of the image, the edge features of the weld were effectively identified, and the centreline of the weld was extracted. For the detection of the offset of the bead geometry, the least squares method could be employed to quickly find the offset of the bead geometry. By comparing the actual measured and calculated offsets, the maximum error is about 2.3 degrees, which proved that the developed algorithm is accurate and reliable. It can be applied for the welding robotic control system which performed welding tasks by the weld centreline identified with the accuracy and automation of welding greatly. 


\section{Acknowledgements}

This work was supported by the Project of Technology Development for Industry Core (No.20002772, Smart Logistics Logistics \& Production Automation System for Pipe Spool, Steel Out- Fitting and Block Logistics in Shipbuilding and Offshore Platform) funded by the Ministry of Trade, Industry \& Energy (MOTIE, Korea).

\section{References}

[1] Manas Kr. Bera, P. S. Lal Priya, B. Bandyopadhyay and A. K. Paul, "A Discrete-time sliding mode control of GMA welding systems using infrequent output measurements," in IEEE 2013 European Control Conference, pp. 3736-3741, 2013.

[2] A. Mital, M. Govindaraju and B. Subramani, "A comparison between manual and hybrid methods in parts inspection," Integrated Manufacturing Systems, vol. 9, no. 6, pp. 344-349, 1998.

[3] Q. X. Wang, B. D. Sun and D. Li, "Research on image processing of the recognitions of seam position," Welding Journal, 2005, pp. 59-63.

[4] Y. Shao, Z. Wang and Y. Zhang, "Monitoring of liquid droplets in laser-enhanced GMA welding," The International Journal of Advanced Manufacturing Technology, vol. 57, pp. 203-214, 2011.

[5] U. V. Koc and K. R. Liu, "Discrete-cosine/sine-transform based motion estimation," in Proceedings of the IEEE International Conference on Image Processing, Austin, TX, vol. 3, pp. 771-775, 1994.

[6] J. Canny, "A computational approach to edge detection," IEEE Trans. on Pattern Analysis and Machine Intelligence, vol. 8, no. 6, pp. 679-698, 1986. 